\title{
Extremely High Energy Neutrinos, Neutrino Hot Dark Matter, and the Highest Energy Cosmic Rays
}

\author{
Shigeru Yoshidal \\ Institute for Cosmic Ray Research, University of Tokyo, Tanashi, Tokyo 188-8502, Japan \\ Günter Sigl and Sangjin Lee \\ Department of Physics \& Astrophysics, Enrico Fermi Institute, The University of Chicago, Chicago, IL 60637-1433
}

(Submitted to Phys.Rev.Lett.)

\begin{abstract}
Extremely high energy $\left(\sim 10^{22} \mathrm{eV}\right)$ cosmic neutrino beams initiate high energy particle cascades in the background of relic neutrinos from the Big Bang. We perform numerical calculations to show that such cascades could contribute more than $10 \%$ to the observed cosmic ray flux above $10^{19} \mathrm{eV}$ if neutrinos have $\sim \mathrm{eV}$ masses. The required intensity of primary neutrinos could be consistent with astrophysical models for their production if the maximum neutrino energy reaches to $\sim 10^{22} \mathrm{eV}$ and the massive neutrino dark matter is locally clustered. Future observations of ultra high energy cosmic rays will lead to an indirect but practical search for neutrino dark matter.
\end{abstract}

95.85.Ry, 95.30.Cq, 98.70.Sa, 98.70.Vc, 95.35.+d

It has been claimed that pure cold dark matter (CDM) leads to a larger baryon fraction $\left(\Omega_{b}\right)$ than predicted by big bang nucleosynthesis (BBN) if the observed hot Xray-emitting gas represents a fair sample of the universe [1]. An admixture of hot dark matter (HDM) with CDM shifts the estimates of the baryon fraction closer to that by BBN. In addition, this mixed cold + hot dark matter model (CHDM) has been shown to agree well with the cosmic microwave background (CMB) spectrum measured by $\mathrm{COBE}$, and galaxy group properties such as the number density of clusters [2]. Neutrinos are the best candidate for HDM and a total neutrino mass of $5 \mathrm{eV}$, or $m_{\nu_{\mu}} \sim m_{\nu_{\tau}} \sim 2.4 \mathrm{eV}\left(\Omega_{\nu} \simeq 0.2\right)$ may be a solution consistent with all available observations.

If HDM consists of cosmological background neutrinos $(\mathrm{CBN})$ with $\sim \mathrm{eV}$ mass and there exist cosmic neutrino beams reaching to $\sim 10^{22} \mathrm{eV}$, the interactions of these extremely high energy (EHE) cosmic neutrinos with the $\mathrm{CBN}$ during their propagation can become significant [3] due to the enhanced interaction probability at the $\mathrm{Z}$ boson resonance. The resulting neutrino cascade causes modifications such as a bump and a dip in the EHE neutrino spectrum at Earth [4]. The cascade contains several hadronic decay channels that produce mostly pions which in turn reproduce neutrinos through their decay [5], but also $\gamma$-rays and some nucleons. Hence this mechanism has been proposed [6,7] as an explanation of the highest energy cosmic rays (HECRs) whose flux above $\sim 5 \times 10^{19}$ $\mathrm{eV}$ is severely attenuated by photopion production on the cosmic microwave background (CMB) [8] in case of nucleon primaries, forming the Greisen-Zatsepin-Kuzmin (GZK) cutoff [9,

The evolution of cascades initiated by EHE cosmic neutrinos is determined by very complex chains of interactions: The neutrinos undergo $\nu \nu$ reactions which involve the exchange of $\mathrm{W}$ and $\mathrm{Z}$ bosons and hadroniza- tion of their strongly interacting decay products. The produced photons, electrons and protons collide with the $\mathrm{CMB}$, the infrared and optical background (IR/O) and the universal radio background (URB) 10,11 initiating electromagnetic cascades. Electrons are also subject to synchrotron cooling in extragalactic magnetic fields (EGMF). The final particle fluxes after propagation depend on all these interactions and solving the relevant transport equations is inevitable for an accurate evaluation of the consequences of this scenario. In this letter we present numerical calculations of the "primary" EHE neutrinos and the "secondary" $\gamma$-rays and protons that may contribute a sizable fraction of the observed HECRs above $\simeq 10^{19} \mathrm{eV}$, under different assumptions concerning neutrino mass and local density enhancement of the HDM. Several observable signatures to confirm or rule out this scenario are discussed followed by a summary.

Cascading Calculation.- Our numerical calculations combine simulation codes for neutrino cascades [4,5], and for electromagnetic cascades and nucleon propagation 10,12. Detailed accounts of these codes are provided in Refs. [4, 12]. The following processes are included: Inelastic and elastic $\nu \nu$ collisions involving an exchange of either a $\mathrm{W}$ or a $\mathrm{Z}$ boson on the CBN; the subsequent decays of produced $\pi$ 's, $\mu$ 's, and $\tau$ 's, hadronization of quark jets, all of which eventually feed into the electromagnetic, neutrino, and nucleon channels; $\gamma \gamma \rightarrow e^{+} e^{-}$on the CMB, the IR/O and the URB; inverse Compton scattering on the same backgrounds; triplet pair production and double pair production on the CMB; synchrotron cooling in the EGMF; the nucleon interactions on the CMB (pair production and photopion production), and neutron decay; redshifting and evolution of the black body temperature due to expansion of the universe. For the IR/O we used recent data [13], and for the (unmeasured) URB we used the highest prediction of Ref. [14] yielding conservatively 
low $\gamma$-ray fluxes around $10^{20} \mathrm{eV}$ for which the URB is the most important target for pair production. We neglect interactions of EHE neutrinos with the CMB photons which are of comparable importance to those with the $\mathrm{CBN}$ only for neutrino energies above the $\mathrm{Z}$ resonance [15.

The hadronic decay of $\mathrm{Z}$ bosons resonantly produced by neutrinos of energy

$$
E_{\mathrm{res}}=M_{z}^{2} / 2 m_{\nu}=4 \times 10^{21}\left(\frac{m_{\nu}}{1 \mathrm{eV}}\right)^{-1} \mathrm{eV}
$$

with the $\mathrm{CBN}$ is the most important neutrino process for production of $\gamma$-rays and nucleons whose spectra are determined by the hadron fragmentation function. At the energy range around the $\mathrm{Z}$ pole, this has been measured accurately by the LEP at CERN. We implemented empirical functions using the MLLA approximation [16] in our code, which have been fitted by measurements of the inclusive production rates of $\pi^{ \pm}$and $p \bar{p}$ with the OPAL detector [17]. This constitutes the major revision of the original codes in Refs. [4, 4 ,

The dominant contribution to secondary particle fluxes from resonant $\mathrm{Z}$ production can be estimated analytically, for example for the produced $\gamma$-ray spectrum,

$$
\left.\left.\frac{d N_{\gamma}}{d E_{\gamma} d L} \sim \sqrt{2} \pi \frac{\Gamma_{Z}}{M_{Z}} \frac{1}{\lambda_{Z}} \frac{d N_{\nu}}{d E_{\nu}}\right|_{E_{\nu}=E_{r e s}} \frac{d n_{h}}{d x}\right|_{x=E_{\gamma} / E_{r e s}},
$$

where $\Gamma_{Z} \simeq 0.03 M_{Z}$ is the decay width of the $\mathrm{Z}$ boson whose mass is $M_{Z}, d n_{h} / d x$ is the $\pi^{0}$ fragmentation spectrum, and $\lambda_{Z} \simeq 38 \mathrm{Gpc}$ is the mean free path of neutrinos at $E_{\text {res }}$ given by Eq. (1). The number of photons above $3 \times 10^{19} /\left(m_{\nu} / \mathrm{eV}\right) \mathrm{eV}$ is then $0.012 E_{\text {res }}\left(d N_{\nu} / d E_{\nu}\right)\left(E_{\text {res }}\right) \mathrm{Mpc}^{-1}$, compared to 0.03 from our full numerical calculation which includes contributions from all channels and uses the more accurate MLLA formula for the fragmentation function.

The particle fluxes.- For a general discussion we consider a homogeneous distribution of sources radiating EHE neutrinos with a constant differential spectrum $\propto E^{-q}$ and a luminosity per comoving volume that scales as $(1+z)^{m}$ between $z=z_{\min }$ and $z=z_{\max }$ [4], with $m$ characterizing source evolution. Because of the small neutrino absorption probability, the results are essentially independent of $z_{\min } \lesssim 1$. We assume a flat universe with a Hubble constant of $H_{0}=65 \mathrm{~km} \mathrm{sec}^{-1}$ $\mathrm{Mpc}^{-1}$ which is consistent with the CHDM picture of the universe [1,2]. We use $q=1$, a typical spectral index expected for neutrinos produced from photopion production by accelerated protons 18 20. Results are, however, rather insensitive to $q$ for $q \lesssim 2$. The ratio of emitted $\nu_{\mu}$ and $\nu_{e}$ fluxes is assumed to be $\simeq 1.86$, as expected from charged pion decay. Furthermore, for the moment we assume that the source luminosity in $\gamma$-rays and nucleons is negligible compared to the neutrino luminosity.

HDM is usually expected to cluster locally and the Fermi distribution with a velocity dispersion $v$ yields an overdensity $f_{\nu} \lesssim v^{3} m_{\nu}^{3} /(2 \pi)^{3 / 2} / \overline{n_{\nu}} \simeq$ $330\left(v / 500 \mathrm{~km} \mathrm{sec}^{-1}\right)^{3}\left(m_{\nu} / \mathrm{eV}\right)^{3}$ over the uniform CBN 21]. If clustering occurs on a scale $l_{\nu}$ smaller than the typical attenuation length $l_{\text {att }} \sim$ few Mpc of nucleons and $\gamma$-rays around $10^{20} \mathrm{eV}$, the ratio of their fluxes produced on that scale to the ones produced on the uniform background is $\simeq f_{\nu} l_{\nu} / l_{\text {att }}$. Therefore, while clustering in the galactic halo or in a nearby galaxy cluster is unlikely to contribute to the HECR flux [22, neutrinos clustering in the local Supercluster may have $f_{\nu} \sim 100, l_{\nu} \sim$ few Mpc.

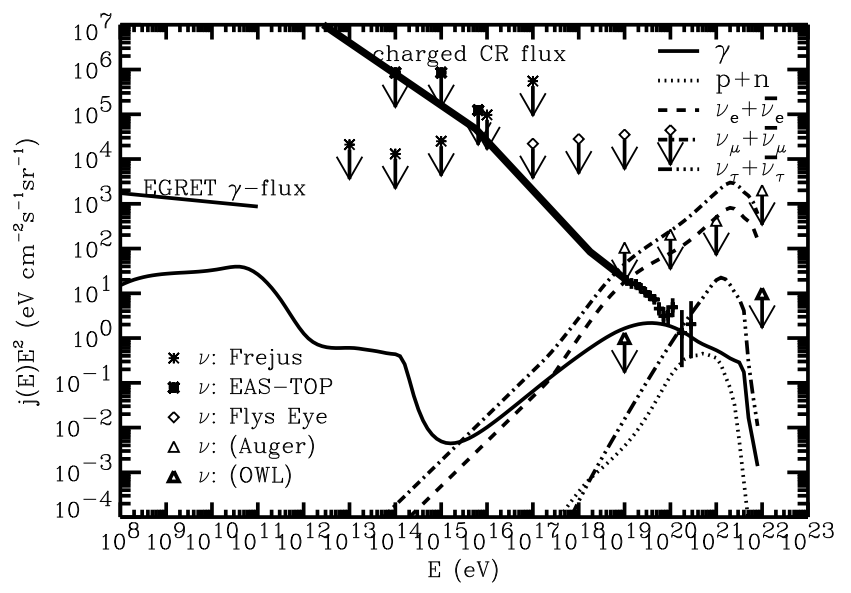

FIG. 1. Energy spectra of nucleons, $\gamma$-rays and neutrinos for the scenario described in the text. Error bars are the combined data from the Fly's Eye 26] and the AGASA 27] experiments above $10^{19} \mathrm{eV}$. Also shown are piecewise power law fits to the observed charged CR flux below $10^{19} \mathrm{eV}$, the EGRET measurement of the diffuse $\gamma$-ray flux between 30 $\mathrm{MeV}$ and $100 \mathrm{GeV}$, and experimental neutrino flux limits from Frejus [24] and Fly's Eye [25], as well as projected neutrino sensitivities of the future Pierre Auger 28] and NASA's OWL [29] projects.

In Fig. 1 1 we show the calculated spectra for the following typical case: $m=3, z_{\min }=0, z_{\max }=3$, an EGMF $\lesssim 10^{-12}$ Gauss, $m_{\nu_{e}}=m_{\nu_{\mu}}=m_{\nu_{\tau}}=1 \mathrm{eV}$, $f_{\nu} \simeq 300$, and $l_{\nu}=5 \mathrm{Mpc}$. It can be seen clearly that the predicted fluxes are consistent with the measurement of the diffuse $\gamma$-ray flux by EGRET 23] and with upper limits on neutrino fluxes by Frejus 24] and Fly's Eye [25]. Typically, the energy content in the produced low energy cascade $\gamma$-rays is a few percent of the neutrino energy which agrees with a rough analytical estimate giving $\sim 10 /\left(H_{0} \lambda_{Z}\right) \Gamma_{Z} / M_{Z}$. By scaling the cosmologically produced low energy $\gamma$-ray flux in Fig. 1 with $l_{\text {att }} /\left(f_{\nu} l_{\nu}\right)$, the EGRET constraint on diffuse $\gamma$-rays requires $f_{\nu} \gtrsim 20\left(l_{\nu} / 5 \mathrm{Mpc}\right)^{-1}$.

The EHE part of the secondary $\gamma$-rays and protons possibly constitute a hard component of the observed HECRs without a GZK cutoff. The energy content in this "visible" HECR component is about $\left(\Gamma_{Z} / M_{Z}\right)\left(f_{\nu} l_{\nu} / \lambda_{Z}\right) E_{\mathrm{res}}^{2}\left(d N_{\nu} / d E_{\nu}\right)\left(E_{\mathrm{res}}\right)$, again consistent with the fluxes shown in Fig. 1. The collisions of the 
EHE cosmic neutrinos with the HDM can be responsible for $\sim 10 \%$ of the observed cosmic rays above $10^{19} \mathrm{eV}$ with dominant contributions of $\gamma$-rays above the GZK cutoff. The fluxes deviate at most by $50 \%$ for $m_{\nu_{e}} \ll m_{\nu_{\mu}}$.

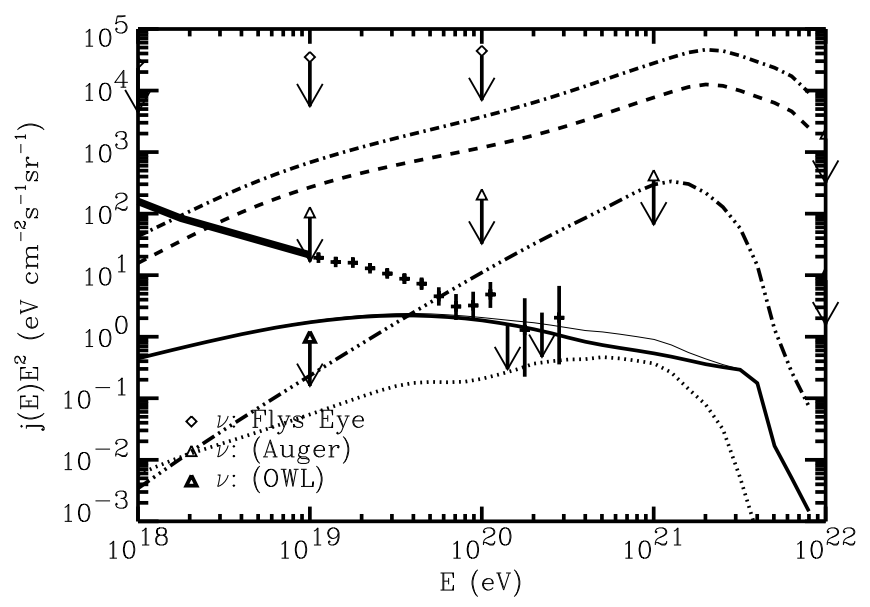

FIG. 2. Same as in Fig. 1 but for the case of an overdensity of 20 over $5 \mathrm{Mpc}$, showing fluxes above $10^{18} \mathrm{eV}$. Line key as in Fig. 11 with the "visible" sum of $\gamma$-ray and nucleon fluxes shown as thin solid line in addition, as well as upper limits in the bins where no HECR were seen below the highest energy event. This case sets an upper bound for the intensity of primary EHE neutrino beams allowed by the EGRET diffuse $\gamma$-ray limit, assuming only secondaries of neutrino interactions contribute to the EGRET flux.

Fig. 2 shows the high energy part of the resultant spectra above $10^{18} \mathrm{eV}$ as in Fig. 1, but for the case of the lower local enhancement of the neutrino dark matter, $f_{\nu}=20$ over a scale $l_{\nu}=5 \mathrm{Mpc}$, the lowest possible $f_{\nu}$ allowed by the EGRET bound. As compared to the case for stronger clustering shown in Fig. 1, the required EHE neutrino intensity is 10 times larger.

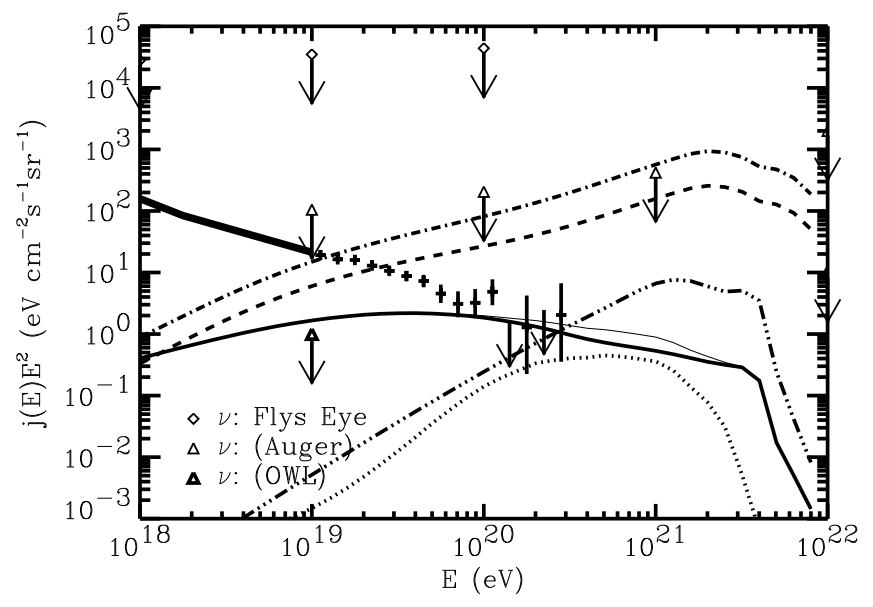

FIG. 3. Same as in Fig. 2 but for the optimistic case of an overdensity of $10^{3}$ over $5 \mathrm{Mpc}$ that would be required if $L_{\gamma} \simeq 13 L_{\nu} / 3$.
In general, models based on photopion production predict an integrated photon source luminosity $L_{\gamma}$ that is comparable to the total neutrino luminosity, $L_{\gamma} \simeq$ $\frac{13}{3} L_{\nu}$ [19,30. In this case, the EGRET constraint translates into the more stringent requirement $f_{\nu} \gtrsim$ $10^{3}\left(l_{\nu} / 5 \mathrm{Mpc}\right)^{-1}$, as can be seen by applying the above mentioned scaling to the integrated neutrino luminosity from Fig. 1. Fig. 3 shows the fluxes for this optimistic case of strong clustering. This bound on $f_{\nu}$ can be relaxed if most of $L_{\gamma}$ does not appear at EGRET energies, but is dominantly released in the $\mathrm{TeV}$ range. This could be a detectable signature from individual point sources [31], in addition to the secondary $\gamma$-rays from neutrino interactions appearing at EGRET energies. Furthermore, the scenario discussed here requires sources that are optically thick for accelerated protons with respect to photopion production because otherwise the observable proton flux below the GZK cutoff would be comparable to the neutrino flux 20].

Discussion.- The EHE neutrino scenario we explored here is quite solid in terms of the particle physics because the interactions with the cosmological backgrounds occur in the well measured LEP energy range. No physics beyond the Standard Model is involved except neutrino mass. The major uncertainty arises in the question whether any astronomical objects are capable of producing neutrinos with energies of $\sim E_{\text {res. }}$. In the conventional models invoking the decay of photoproduced pions, primary protons must be accelerated to $\sim 20 E_{\text {res }} \sim$ $10^{23} /\left(m_{\nu} / \mathrm{eV}\right) \mathrm{eV}$ in order to generate neutrinos of energy $E_{\text {res }}$. Furthermore, the sources would need a dense photon target to supply high neutrino luminosity and to absorb protons and $\gamma$-rays. Thus, a new model for the neutrino beam sources may be necessary [32].

Interestingly, the energy generation rate of $\sim E_{\text {res }}$ neutrinos for the scenario shown in Fig. 11, $1.8 \times 10^{45}$ erg $\mathrm{Mpc}^{-3} \mathrm{yr}^{-1}$ divided by the the rate of cosmological Gamma Ray Bursts (GRBs), $3 \times 10^{-8} \mathrm{Mpc}^{-3} \mathrm{yr}^{-1}$ [33], yields $\sim 6 \times 10^{52} \mathrm{erg}$, and is comparable to the observed energy release including afterglow from a typical GRB in the BATSE range [34].

The EHE neutrino scenario has several advantages to explain the HECR observation. The observed relatively hard spectrum without GZK cutoff 27] is reasonably reconstructed in our model which is determined mainly by the well-measured hadron fragmentation function at the $\mathrm{Z}$ pole and the energy loss process in the cosmological backgrounds, regardless of the nature of the EHE neutrino sources. The highest energy events above the GZK cutoff can originate from very distant powerful objects because neutrinos propagate without significant energy loss. For example, the AGN 3C147 at a redshift of 0.545 is a candidate for the Fly's Eye $3 \times 10^{20} \mathrm{eV}$ event [35]. For the same reason, it is natural that we found no nearby powerful astronomical objects in directions of the possible event clusters observed by AGASA [36]. Because 
the EHE neutrino beams can be responsible for a sizable fraction of cosmic rays above $10^{19} \mathrm{eV}$, this scenario can explain the observational fact that the intensity of the events observed above the GZK cutoff is consistent with the extrapolation of the flux from lower energies.

Among the observable signatures of the neutrino scenario are the primary EHE neutrinos whose flux should be detectable, as projected sensitivities of future experiments such as the Pierre Auger [28] and NASA's OWL [29] projects suggest. Correlation of the arrival direction of the EHE neutrino and the secondary HECR showers may also be observable. As opposed to conventional models with nucleon primaries, our model predicts that some of the observed HECRs should originate in sources at cosmological distances. At energies beyond the GZK cutoff, the correlation of arrival directions of HECR showers with sources at distances $\gg l_{\text {att }}$ should be easy to detect since the background from a conventional nucleon component should be suppressed due to the GZK effect, whereas the component proposed here continues as a relatively flat spectrum. Finally, this scenario predicts a $\gamma$-ray domination above $10^{20} \mathrm{eV}$, and next generation experiments should settle the question whether observed HECR are consistent with $\gamma$-ray primaries [35].

In summary, we have seen that collisions of EHE cosmic neutrino beams with $\sim \mathrm{eV}$ mass neutrino dark matter would explain the observed HECR energy spectrum, regardless of the nature of the neutrino sources if the maximum neutrino energy reaches to the $\mathrm{Z}$ boson pole region and the dark matter is clustered on the Supercluster scale by amounts consistent with expectations. Although EHE neutrino sources require very high efficiency of converting the energy to neutrino flux which may require a new production mechanism of neutrinos, the necessary neutrino intensity can be consistent with observed diffuse $\gamma$-ray fluxes and the GRB energy release rate. The EHE neutrino scenario is a way of producing a relatively flat component of nucleons and $\gamma$-rays that provides a significant fraction of the HECR flux above $10^{19}$ $\mathrm{eV}$, dominating above the GZK cutoff, without invoking physics beyond the Standard Model except neutrino mass. Future observations of HECRs lead to indirect search for signatures of neutrino dark matter.

We thank Eli Waxman and Makoto Sasaki for helpful discussions and advice and Tom Weiler and Pijush Bhattacharjee for encouragement. Paolo Coppi is acknowledged for earlier collaboration on electromagnetic cascades. At the University of Chicago this research was supported by the DoE, NSF, and NASA.

* $\quad$ Electronic Address: syoshida@icrr.u-tokyo.ac.jp
[1] R. W. Strickland and D. N. Schramm, Astrophys. J. 481, 571 (1997).

[2] J. R. Primack et al., Phys. Rev. Lett. 74, 2160 (1995).

[3] E. Roulet, Phys. Rev. D 47, 5247 (1993).

[4] S. Yoshida et al., Astrophys. J. 479, 547 (1997).

[5] S. Yoshida, Astropart. Phys. 2, 187 (1994).

[6] T. J. Weiler, hep-ph/9710431, to be published in Astropart. Phys.

[7] D. Fargion, B. Mele, and A. Salis, astro-ph/9710029.

[8] For reviews, see S. Yoshida and H. Dai, J. Phys. G 24, 905 (1998).

[9] K. Greisen, Phys. Rev. Lett. 16, 748 (1966); G. T. Zatsepin and V. A. Kuzmin, JETP Lett. 4, 178 (1966).

[10] G. Sigl et al., Phys. Lett. B 392, 129 (1997).

[11] R. J. Protheroe and T. Stanev, Phys. Rev. Lett. 77, 3708 (1996); erratum Phys. Rev. Lett. 78, 2420 (1997).

[12] S. Lee, Phys. Rev. D 58, 043004 (1998).

[13] D. J. Fixsen et al., Astrophys. J. 490, 482 (1997).

[14] R. J. Protheroe and P. L. Biermann, Astropart. Phys. 6, 45 (1996).

[15] D. Seckel, Phys. Rev. Lett. 80, 900 (1998).

[16] V. A. Khoze and W. Ochs, Int. J. Mod. Phys. A 12, 2949 (1997).

[17] R. Akers et al., Z. Phys. C63, 181 (1994).

[18] F. W. Stecker and M. H. Salamon, Space. Sc. Rev. 75, 341 (1996).

[19] F. Halzen and E. Zas, Astrophys. J. 488, 669 (1997).

[20] E. Waxman and J. Bahcall, hep-ph/9807282, submitted to Phys. Rev. D.

[21] see, e.g., P. J. E. Peebles, Principles of Physical Cosmology, Princeton University Press, New Jersey, 1993.

[22] E. Waxman, astro-ph/9804023, submitted to Astropart. Phys.

[23] P. Sreekumar et al., Astrophys. J. 494, 523 (1998).

[24] W. Rhode et al., Astropart. Phys. 4, 217 (1996).

[25] R. M. Baltrusaitis et al., Phys. Rev. D 31, 2192 (1985).

[26] D. J. Bird et al., Phys. Rev. Lett. 71, 3401 (1993).

[27] M. Takeda et al. Phys. Rev. Lett. 81, 1163 (1998).

[28] K. S. Capelle, J. W. Cronin, G. Parente, and E. Zas, Astropart. Phys. 8, 321 (1998).

[29] J. F. Ormes et al., in Proc. 25th International Cosmic Ray Conference (Durban, 1997), eds.: M. S. Potgieter et al.

[30] K. Mannheim, Astropart. Phys. 3, 295 (1995).

[31] F.A. Aharonian, P.S.Coppi, and H.J.Völk, Astrophys.J. 423, L5 (1994).

[32] One possible mechanism to produce neutrinos efficiently without EHE proton radiation is the collision of protons directly accelerated by Wakefield and Snowplow plasma at initial GRB fireball with thick $\gamma$-rays. See Y. Takahashi in Proceedings of Workshop on Observing Giant Air Showers from Space, edited by J. F. Krizmanic et al. (The American Institute of Physics, 1998), p. 469.

[33] S. Mao and B. Paczyński, Astrophys. J. 388, L45 (1992); E. Cohen and T. Piran, Astrophys. J. 444, L25 (1995).

[34] E. Waxman and J. Bahcall, Phys. Rev. Lett. 78, 2292 (1997) and references therein; M. Vietri, Phys. Rev. Lett. 80, 3690 (1998).

[35] J. W. Elbert and P. Sommers, Astrophys. J. 441, 151 (1995); F. Halzen et al., Astropart. Phys. 3, 151 (1995).

[36] N. Hayashida et al., Phys. Rev. Lett. 77, 1000 (1996). 